\title{
SCREENING E AVALIAÇÃO ANTIFÚNGICA DO HIDROLATO E DO ÓLEO ESSENCIAL DE MENTHA PIPERITA
}

\author{
SCREENING AND ANTIFUNGAL EVALUATION OF HYDROLATE AND ESSENTIAL \\ OIL MENTHA PIPERITA
}

\author{
Évellin do Espirito Santo ${ }^{1 *}$, Rafael Boffo de Souza ${ }^{2}$, Elizandra Hertel Lenhardt ${ }^{3}$, Silvia \\ Toledo Talarico 4 , Marina de Freitas Silva ${ }^{5}$
}

1 - Mestranda do programa de pós-graduação em tecnologia bioquímico - farmacêutica - Universidade de São Paulo (USP) - São Paulo/SP.

2 - Mestrando do programa de pós-graduação em química fundamental - Universidade de São Paulo (USP) - São Paulo/SP.

3 - Docente do curso de enfermagem - Universidade Estadual do Mato Grosso (UNEMAT) - Diamantino/MT.

4 - Docente do curso de farmácia - Faculdade Oswaldo Cruz (FOC), São Paulo/SP.

5 - Docente do curso de farmácia - Universidade Paulista (UNIP), São Paulo/SP.

\section{RESUMO:}

O óleo essencial de Mentha piperita L é amplamente utilizado nas indústrias alimentícia, farmacêutica e de cosméticos. Os tradicionais processos de obtenção deste tipo de óleo geram como subproduto o hidrolato, uma complexa mistura de compostos orgânicos em meio aquoso, que é considerado um efluente da extração. Avaliaram-se propriedades físico-químicas e antifúngicas do óleo essencial e do hidrolato frente a um "pool" de microrganismos, caracterizados macro e microscopicamente como leveduras, provenientes de morangos do comércio da cidade de São Paulo, SP. O óleo essencial e o hidrolato foram obtidos pelo método de Cleavenger por hidrodestilação. A avaliação da atividade antifúngica do hidrolato, do óleo essencial e de uma mistura óleo essencial e hidrolato (1:1) foram realizadas empregando-se o do método de difusão em discos. O hidrolato apresentou características físico-químicas intermediárias às da água destilada e do óleo essencial. Quanto à análise da inibição do "pool" de contaminantes fúngicos oriundos de morangos, verificou-se que os microrganismos apresentaram sensibilidade frente ao óleo essencial e à mistura de hidrolato-óleo (1:1). Nos testes realizados utilizando apenas hidrolato não foram observados halos de inibição contra os microrganismos avaliados. A utilização do hidrolato em conjunto com o óleo essencial de Mentha piperita $L$ mostra-se promissora frente ao combate de leveduras com importância na conservação de alimentos, tal como o foi em morangos, permitindo assim a reutilização do resíduo de extração (hidrolato) associado ao óleo essencial, em alternativa à aplicação de fungicidas sintéticos, agregando valor a este efluente.

PALAVRAS-CHAVES: leveduras, conservantes, alimentos, hortelã pimenta, menta.

\section{ABSTRACT:}

The essential oil of Mentha piperita $L$ is widely used in the food, pharmaceutical and cosmetics industries. The traditional processes of attainment this type of oil generate, as a by-product the hydrolate, a complex mixture of organic compounds in the aqueous medium, which is considered an extraction effluent. Physical-chemical properties and antifungal activity of essential oil and hydrolate were evaluated, against a "pool" of microorganisms, 
characterized macro and microscopically as yeasts, from of strawberries in the trade of the city of São Paulo, SP. The essential oil and hydrolate were obtained by the hydrodistillation cleavage method. An evaluation of the antifungal activity of hydrolate, essential oil and a mixture of essential oil and hydrolate (1:1) was performed using the disc diffusion method. The hydrolate has intermediate physical-chemical characteristics to distilled water and essential oil. As for the analysis inhibition of "pool" of contaminants from strawberries, it was found that the microorganisms detected sensitivity to the essential oil and the hydrolate oil mixture (1:1). In the tests carried out using only hydrolate, inhibition halos were not observed against the microorganisms. The use of hydrolate in conjunction with the essential oil of Mentha piperita $L$. is promising in the face of combating yeasts with importance in food preservation, as it was in strawberries, thus allowing the reuse of the extraction residue (hydrolate) essential oil, as an alternative to the application of synthetic fungicides, adding value to this effluent.

KEYS: yeasts, preservative, food, peppermint, mint.

\section{INTRODUÇÃO}

A família Lamiaceae abriga grande abundância de espécies aromáticas, sendo boa parte destas encontradas no gênero Mentha (GUPTA et al., 2017). O Brasil se configura entre os grandes produtores mundiais de plantas deste gênero e a extração de óleos essenciais se torna uma atividade econômica de grande relevância (KALEMBA; SYNOWIEC, 2019; GUPTA et al., 2017).

O óleo essencial de Mentha piperita (menta ou hortelã-pimenta) é amplamente utilizado nas indústrias de alimentos, farmacêutica e de cosméticos, desempenhando funções bactericida, fungicida e outras mais atividades biológicas, agregando valor a este produto (MACHIANI et al., 2018).

Os óleos essenciais são misturas complexas e altamente variáveis de compostos voláteis, oriundos do metabolismo secundário vegetal. De forma geral, são constituídos por terpenóides e compostos aromáticos, tendo sua composição química afetada por fatores abióticos, de natureza quimiotípica e em função da espécie escolhida (MONTEIRO, 2009). Em especial, o óleo essencial de M. piperita é caracterizado pela presença de monoterpenos em grandes proporções: mentol (20-60\%); seguido de seu precursor, mentona (14-32\%); e baixos teores de pulegona (<4\%), mentofurano (1-9\%) e acetato de mentila (2,8-10\%), proporcionando boa qualidade e afetando de modo significativo o seu valor comercial (BEHN et al., 2010; COSTA et al., 2012).

A extração de óleos essenciais, em grande parte, é realizada utilizando o vapor d' água como agente carreador, por meio das técnicas de hidrodestilação e de extração por arraste a vapor (SOLANKI; DESAI; PARIKH, 2019). Após a separação do óleo propriamente dito, o resultante é um excedente de uma solução aquosa contendo componentes 
hidrossolúveis e voláteis da planta, o qual recebe a designação de hidrolato, sendo este, considerado um efluente (RAJESWARA RAO et al., 2016). Devido à natureza diluída de sua composição, a aplicação do hidrolato torna-se altamente tolerável, apresentando atividades anti-inflamatórias e antissépticas (HOARE; WILSON, 2010).

Anualmente a indústria alimentícia sofre perdas econômicas devido ao descarte de alimentos deteriorados durante o processamento e a manipulação (MEDEIROS et al., 2015; SOUZA et al., 2017). Em sua maioria, tal degradação está relacionada à contaminação por microrganismos como leveduras, fungos filamentosos e bactérias, os quais geram toxinas e micotoxinas que produzem sabores e odores desagradáveis, inviabilizando o consumo dos alimentos (MEDEIROS et al., 2015).

O objetivo deste trabalho foi a avaliação do potencial antimicrobiano do óleo essencial e do hidrolato de Mentha piperita obtidos por hidrodestilação, empregando um método de avaliação antifúngica in vitro, visando a preservação de frutas in natura. Para isto, realizou-se a análise das propriedades físico-químicas dos produtos de destilação (óleo essencial e hidrolato), possibilitando a determinação das características dos potenciais agentes de inibição.

\section{MATERIAL E MÉTODOS}

\subsection{Obtenção do material vegetal}

A coleta da Mentha piperita foi realizada em 19/08/2017 às 11 horas da manhã em uma pequena propriedade de cultivo, situada na região da zona leste de São Paulo (233'34.7'S 46³0'30.1'W). A planta foi identificada na Universidade Paulista (UNIP) confrontando as características observadas com a descrição macroscópica detalhada na Farmacopeia Brasileira (ANVISA, 2019), onde foram coletadas suas folhas, lavadas com água destilada e secas ao ar a $24 \stackrel{\circ}{ } \mathrm{C}$, por um período de 24 horas. Depois de seco, o material vegetal foi aliquotado em 20 amostras de $50 \mathrm{~g}$ cada, destinadas ao processo de extração.

\subsection{Hidrodestilação}

A obtenção do óleo essencial, juntamente com o seu hidrolato correspondente, foram obtidos através do método de extração de Cleavenger. Uma amostra de $50 \mathrm{~g}$ de folhas de hortelã (Mentha piperita) foi acondicionada em um balão de fundo redondo com 
capacidade volumétrica de $1 \mathrm{~L}$, e adicionado 0,5 $\mathrm{L}$ de água destilada. Utilizando-se de manta aquecedora, foi produzido vapor d'água que ao passar pelo condensador, promoveu o carreamento dos compostos voláteis presentes nas folhas. A mistura de água e óleo foi resfriada e recolhida em seu estado líquido; o processo foi repetido por 20 vezes (BEIJI; TORKI-HARCHEGANI; PIRBALOUTI, 2018).

\subsection{Caracterização físico-química do hidrolato e do óleo essencial}

As análises físico-químicas como pH, tensão superficial, osmolaridade e densidade foram realizadas em triplicata, utilizando água destilada como padrão para a comparação dos resultados.

\subsubsection{Determinação do $\mathrm{Ph}$}

$\mathrm{O} \mathrm{pH}$ foi determinado com o auxílio de pHmetro digital (pH210 Colleman, Santo André, SP, Brasil), previamente calibrado com soluções-tampão pH 4,0 e 7,0.

\subsubsection{Determinação da tensão superficial}

Determinou-se a tensão superficial das amostras em tensiômetro (Modelo K9, Hruss, GmbH, HB, Alemanha), com um anel descrito por Nuoy, 1925. Os testes foram realizados em triplicata utilizando $15 \mathrm{~mL}$ de amostra em um recipiente de vidro, onde o anel de platina foi submerso e após o desprendimento do mesmo da superfície da amostra, obteve-se as medições.

\subsubsection{Determinação da osmolaridade}

A osmolaridade foi determinada por osmômetro (Osmomat 030, OttendorfOkrilla, Alemanha) através do ponto de congelamento da solução, sendo calibrado com solução de $\mathrm{NaCl} 300$ Milliosmol/kg.

\subsubsection{Cálculo da densidade}

A densidade foi obtida pelo método picnométrico, conforme descrito na Farmacopéia Brasileira (ANVISA, 2019). A cada volume fixo (5 mL) dos líquidos amostrados (hidrolato e óleo essencial), ambos à temperatura de $20^{\circ} \mathrm{C}$, obteve-se as respectivas massas em uma 
balança analítica. Destas, depois de subtraído a massa do picnômetro vazio, o cálculo da densidade relativa $\left(d_{20}^{20}\right)$ foi obtido da razão entre as massas das amostras líquidas e a massa de água destilada, nas mesmas condições experimentais. Com este valor, a densidade de massa $\left(\rho_{\mathrm{m}}\right)$ à $20^{\circ} \mathrm{C}$, foi calculado pela equação $(1)$ :

$$
d=\frac{m}{v}
$$

\subsection{Meio de cultivo utilizado e "pool" de contaminantes}

O meio Sabouraud Dextrose Agar (SDA, Neogen Europe Ltd., Lesher Place, Lansing, Estados Unidos) foi preparado de acordo com as especificações do fabricante.

Foram adquiridos morangos in natura em uma feira livre da cidade de São Paulo SP e mantidos à temperatura próxima de $21^{\circ} \mathrm{C}$ em laboratório com termo-higrômetro digital, durante 8 dias. Após este período, com a descaracterização do aspecto inicial da fruta, os microrganismos foram coletados da região externa do vegetal com o auxílio da alça de Drigalski e repicados em placa de Petri contendo meio SDA. Para a identificação da presença de leveduras, foram consideradas características microscópicas como formas lenticulares e hifas através da análise em microscópio óptico (Olympus BX51), mediante visualização após coloração de Gram.

\subsection{Avaliação antifúngica do óleo essencial e do hidrolato}

Foram avaliados o óleo essencial, hidrolato e uma solução na proporção 1:1 de óleo essencial e hidrolato, quanto à eficácia em relação à atividade antifúngica. $A$ análise comparativa foi realizada através do método de difusão em disco. Cada teste foi realizado utilizando dois discos de papel de filtro (5 mm de diâmetro) embebidos em $20 \mu \mathrm{L}$ de cada uma das amostras e colocados sobre o meio SDA, em uma placa de Petri baseado em Ostrosky e colaboradores (2008). As amostras foram incubadas a $37^{\circ} \mathrm{C}$, e durante os intervalos de 24, 44 e 144 horas foram realizadas as medições dos diâmetros dos halos de inibição ao redor de cada disco, com o auxílio de um paquímetro.

\subsection{Análise comparativa dos resultados}

A capacidade de inibição de crescimento do microrganismo frente à aplicação das três amostras foi avaliada tomando-se como parâmetro comparativo a fração entre a área de inibição ao redor de cada halo e a área total da superfície da placa de Petri $(9 \mathrm{~cm}$ de 
diâmetro).

\section{RESULTADOS E DISCUSSÃO}

\subsection{Caracterização físico-química}

O processo de extração utilizando a técnica de hidrodestilação resultou em uma mistura heterogênea bifásica, com o óleo essencial de Mentha piperita (fase superior) obtido como um líquido de coloração ligeiramente amarelada e com odor característico de menta, totalizando um volume de $6,1 \mathrm{~mL}$ (óleo bruto) e rendimento de $0,55 \%(\mathrm{~m} / \mathrm{m}$ ), tomando-se como parâmetro a densidade calculada de 0,9088 g.cm-3 (Tabela 1). Os óleos essenciais são obtidos por hidrodestilação da planta fresca ou parcialmente seca, com um rendimento de 0,3 a 0,7\% (KALEMBA; SYNOWIEC, 2019). Estudos mostraram que ocorre uma variação significativa no teor de óleo essencial quando a colheita do material vegetal é efetuada ao longo do dia, resultando em melhores rendimentos em ocasiões onde a obtenção da planta ocorreu entre o período da manhã e as primeiras horas da tarde (OLIVEIRA et al., 2012; SOUZA et al., 2006). Neste sentido, a maior parte da produção de óleos essenciais está associada ao aumento da incidência de radiação solar e a taxa fotossintética, afetando diretamente a biossíntese de compostos do metabolismo secundário (MARCHESE; FIGUEIRA, 2005).

Tabela 1. Propriedades físico-químicas do óleo essencial, hidrolato e água destilada (padrão).

\begin{tabular}{lccc}
\hline $\begin{array}{l}\text { Propriedades } \\
\text { físico-químicas }\end{array}$ & Óleo essencial & Hidrolato & $\begin{array}{c}\text { Água destilada } \\
\text { (Padrão) }\end{array}$ \\
\hline $\mathrm{pH}$ & $5,71( \pm 0,03)$ & $6,76( \pm 0,07)$ & $6,90( \pm 0,01)$ \\
\hline $\begin{array}{l}\text { Tensão superficial } \\
(\mathrm{mN} / \mathrm{m})\end{array}$ & $28,80( \pm 0,13)$ & $46,30( \pm 0,19)$ & $70,10( \pm 0,11)$ \\
\hline $\begin{array}{l}\text { Osmolaridade } \\
(\mathrm{mOsm} / \mathrm{L})\end{array}$ & Não cristaliza & $\begin{array}{c}0,006 \\
( \pm 0,001)\end{array}$ & 0,000 \\
\hline $\begin{array}{l}\text { Densidade } \\
\left(\text { g.cm }{ }^{-3}\right)(20 \stackrel{\circ}{\circ} \mathrm{C})\end{array}$ & 0,9088 & 1,0516 & $0,001)$ \\
\hline Estado Físico $\left(20^{\circ} \mathrm{C}\right)$ & Líquido & Líquido & Líquido \\
\hline
\end{tabular}

Fonte: próprio autor. 
Da avaliação da composição química do óleo essencial de menta extraído por hidrodestilação, realizada por Oliveira e colaboradores (2012), estes encontraram uma grande variação no teor dos compostos voláteis ao longo do dia, ao passo que no período de ascensão da radiação fotossinteticamente ativa (11h), a composição mostrou-se mais complexa. Os constituintes monoterpênicos oxigenados são obtidos em maiores quantidades (86,54 - 91,32\%), diferindo na proporção dos compostos majoritários segundo as condições de cultivo e natureza quimiotípica. Em estudo semelhante, Souza et al. (2006) encontraram concentrações de 34-42\% de mentona, $24-30 \%$ de mentofurano, $14-22 \%$ de pulegona, 5-8\% de mentol, 3-7\% de acetato de mentila, $2-3 \%$ de 1,8-cineol e $0,6-7 \%$ de limoneno.

Com resultados semelhantes, porém utilizando uma variedade de menta distinta da utilizada anteriormente (Mentha arvensis L.) e sob condições variadas do processo de extração (tempo, temperatura, tamanho da partícula e método de extração), Watanabe e colaboradores (2006) encontraram os principais componentes deste tipo de óleo como sendo mentol, acetato de mentila, mentona, isomentona e limoneno, respectivamente nesta ordem de abundância.

Os valores de $\mathrm{pH}$ obtidos para o óleo essencial de menta $(5,71)$ e para o hidrolato $(6,76)$ indicam soluções de caráter ácido, tendo esta última, características muito semelhantes às da água destilada (TABELA 1).

A utilização de água aquecida como agente extrator possibilita operar com grandes volumes de extração, mas possui a desvantagem de permitir a solubilização de compostos como fenóis, álcoois e ácidos (WATANABE et al., 2006), alterando as propriedades da água de condensação. De fato, a grande quantidade de água em ebulição em contato direto com a pequena fração de óleo essencial, pode perturbar as condições de equilíbrio (éster + água $\square$ ácido + álcool) e ocasionar a hidrólise de ésteres, acidificando o meio (STERRETT, 1962).

Em sua maioria, os compostos orgânicos presentes nos óleos essenciais mostramse pouco solúveis em meio aquoso, sendo praticamente insolúveis no caso de hidrocarbonetos terpênicos e sesquiterpênicos, e dissolvidos de 0,05 a 1,0\% para compostos oxigenados: ésteres; cetonas; aldeídos; fenóis; etc. (FLEISHER; FLEISHER, 1991). Este fato tende a explicar os valores próximos medidos para osmolaridade e densidade do hidrolato (0,006 mOsm.L-1 e 1,0516 g.cm-3) e água destilada (0,000 mOsm.L-1 e 0,9984 g.cm-3), refletindo a natureza semelhante das duas substâncias quanto à presença de solutos em solução. Por se tratar de uma medição por efeito crioscópico, em 
comparação com o ponto de congelamento de uma solução salina $\left(-1,858{ }^{\circ} \mathrm{C}\right)$, não foi possível a medição da osmolaridade para o óleo essencial, pois este não cristalizou.

$\mathrm{Na}$ comparação entre os valores de tensão superficial para as três amostras, verifica-se que 0 hidrolato apresenta medida de tensão superficial $(46,3 \mathrm{mN} / \mathrm{m})$ aproximadamente intermediária entre aqueles registrados para a água destilada (70,1 $\mathrm{mN} / \mathrm{m})$ e óleo essencial $(28,8 \mathrm{mN} / \mathrm{m})$. De fato, este efeito poderia estar relacionado à presença de uma pequena fração do óleo, de menor densidade $(0,9088$ g.cm-3), dispersa sobre a superfície do hidrolato sob a forma de gotículas.

\subsection{Avaliação inibitória do crescimento microbiano}

Após 8 dias foram observadas colônias microbianas de aspecto algodonoso e de coloração preta e branca na superfície dos morangos. Destas, foram retiradas amostras e cultivadas em meio SDA.

As colônias obtidas através do repique em SDA apresentaram coloração branca, com aspecto brilhante e textura cremosa espalhada sobre o meio de cultura. No presente trabalho foi utilizada a temperatura de $37^{\circ} \mathrm{C}$ para a incubação das placas. Segundo VanUden (1985), de fato uma grande variedade de leveduras é capaz de crescer nestas condições.

O método de Gram resultou na uniformidade da coloração roxa dentre as células coradas, e a presença de formas lenticulares foi observado na microscopia.

A coloração de Gram é comumente usada para detectar bactérias em amostras clínicas, mas também pode corar outros organismos, como os fungos. Em uma análise mais detalhada em relação à discriminação de elementos fúngicos, é possível fazer a distinção entre grupos de organismos em específico (MOHAN, 2004; ANVISA, 2004).

Deste modo, os testes de inibição foram realizados com um "pool" de contaminantes, preferencialmente, em sua maioria leveduras. O meio SDA utilizado para o cultivo de leveduras, fungos patogênicos e comensais, permite a seletividade desses microrganismos devido ao pH ácido da formulação de aproximadamente 5,6 $( \pm 0,2)$ e a alta concentração de dextrose (JARRETT; SONNENWIRTH, 1980; HARE, 2012) de 40 g.L-1.

A partir dos halos inibitórios pôde-se avaliar o potencial antifúngico do óleo essencial, da mistura 1:1 de óleo essencial e hidrolato, e apenas do hidrolato frente ao agente contaminante (Tabela 2). Em 24 horas observou-se o mesmo potencial inibitório tanto para o óleo essencial puro quanto para a mistura (1:1), de tal forma que houve 
completa inibição do crescimento do microrganismo sobre a placa de Petri (ausência de halo de inibição). Enquanto que a utilização do hidrolato não acarretou inibição satisfatória (diâmetro do halo de inibição de 0,6 cm), obtendo-se crescimento praticamente semelhante ao da placa controle, sobre a qual, logo no primeiro dia, houve o crescimento na totalidade da superfície avaliada.

Tabela 2. Avaliação da amplitude de inibição do "pool" de contaminantes frente à utilização de $20 \mu \mathrm{L}$ de óleo essencial, mistura de óleo essencial e hidrolato (1:1), e hidrolato em $24 \mathrm{~h}$, $48 \mathrm{~h}$ e $144 \mathrm{~h}$ de incubação a $37^{\circ} \mathrm{C}$.

\section{Inibição calculada por tempo de incubação}

$24 \mathrm{~h} \quad 48 \mathrm{~h} \quad 144 \mathrm{~h}$

Amostras

\begin{tabular}{|c|c|c|c|c|c|}
\hline $\begin{array}{c}\text { Diâmetro } \\
\text { médio do halo } \\
\text { de inibição } \\
\text { (cm) }\end{array}$ & $\begin{array}{l}\text { Área inibida } \\
\text { da placa } \\
(\%)\end{array}$ & $\begin{array}{l}\text { Diâmetro } \\
\text { médio do halo } \\
\text { de inibição } \\
\text { (cm) }\end{array}$ & $\begin{array}{c}\text { Área inibida } \\
\text { da placa } \\
(\%)\end{array}$ & $\begin{array}{c}\text { Diâmetro médio } \\
\text { do halo de } \\
\text { inibição }(\mathrm{cm})\end{array}$ & $\begin{array}{c}\text { Área inibida } \\
\text { da placa } \\
\text { (\%) }\end{array}$ \\
\hline
\end{tabular}

\begin{tabular}{|c|c|c|c|c|c|c|}
\hline Óleo essencial & - & 100 & 3,8 & 42 & 2,9 & 32 \\
\hline \multicolumn{7}{|l|}{ Mistura de óleo } \\
\hline essencial e & - & 100 & 3,1 & 34 & 1,9 & 21 \\
\hline \multicolumn{7}{|l|}{ hidrolato (1:1) } \\
\hline Hidrolato & 0,6 & 6,7 & 0 & 0 & 0 & 0 \\
\hline Placa controle & 0 & 0 & 0 & 0 & 0 & 0 \\
\hline
\end{tabular}

Fonte: próprio autor.

Franzener e colaboradores (2007) verificaram que o aumento da concentração de compostos bioativos presentes no hidrolato, provenientes do óleo essencial, proporcionaria maior inibição do crescimento bacteriano. Assim, os resultados obtidos neste trabalho sugerem que uma relação semelhante de concentração poderia inibir contaminantes fúngicos, já que os resultados da mistura de óleo essencial e hidrolato (1:1) (Tabela 2) apontam neste sentido.

Contudo, o hidrolato proveniente diretamente do processo de hidrodestilação dificilmente conterá quantidade suficiente destes compostos para ser utilizado como 
antimicrobiano. Isso porque os principais constituintes dos óleos essenciais são apenas parcialmente solúveis em meio aquoso (FLEISHER; FLEISHER, 1991).

Assim como estão presentes em quantidades significativas em óleos essenciais de menta (SOUZA et al., 2006; WATANABE et al., 2006), mentona e mentol são os principais componentes que exibem alta atividade antimicrobiana contra bactérias Gram-positivas e Gram-negativas, leveduras e fungos (MAHBOUBI; KAZEMPOUR, 2014). Em um estudo fitoquímico, o extrato aquoso de Mentha piperita apresentou resultado negativo para terpenoides, esteroides, fenóis, flavonoides e alcaloides, sendo positivo apenas para taninos (SINGH; SHUSHNI; BELKHEIR, 2015). Contrapondo a atividade antisséptica do hidrolato, comentada por Hoare e Wilson (2010).

De acordo com Carretto et al. (2010), o óleo essencial de Mentha piperita exibiu atividade antifúngica contra C. albicans, C. tropicalis, C. glabrata e C. parasilosis, mas sua infusão (solução aquosa) não apresentou atividade antifúngica. $O$ alto teor de monoterpenóides oxigenados (mentol e mentona), também encontrados em $\mathrm{M}$. arvensis, revelou atividade antifúngica contra Fusarium solani, Aspergillus niger, Botryodiplodia theobromae, Mucor mucedo e Rhizopus solani (ANWAR et al., 2019).

Como obteve-se, em $24 \mathrm{~h}$ de incubação, placas de amostras de óleo essencial e da mistura de hidrolato-óleo totalmente inibidas, foram calculadas porcentagens de atividade inibitória mínima para melhor compreender qual era a ação das amostras frente ao "pool" de contaminantes em relação ao tempo. Já que não seria possível considerar o primeiro halo.

Sendo assim, conforme os resultados apresentados na Tabela 2 observou-se que na amostra de óleo essencial, sua atividade inicial foi considerando de $100 \%$ em $24 \mathrm{~h}$, o que demonstrou o impedimento do crescimento do microrganismo em toda a extensão da placa. Em 48h, a inibição do microrganismo impactou na inibição de $42 \%$ da área da placa com um halo de $3,8 \mathrm{~cm}$, e $32 \%$ da área foi inibida em $144 \mathrm{~h}$ com um halo de 2,9 cm, no qual observou-se o decaimento da atividade inibitória em relação ao tempo, comparado ao diâmetro do halo de inibição. Da mesma forma, seguiu-se o decréscimo da atividade inibitória da mistura hidrolato-óleo, cuja ação inicial foi de 100\%, demonstrando que nenhuma colônia de microrganismo foi capaz de crescer na placa, de modo que decorrido um período de 48h, a inibição caiu para 34\%, com um halo de diâmetro de $3,1 \mathrm{~cm}$, atingindo valores de $21 \%$ em 144h, com halo de 1,9 cm, comparado ao halo de inibição (Figura 1). 

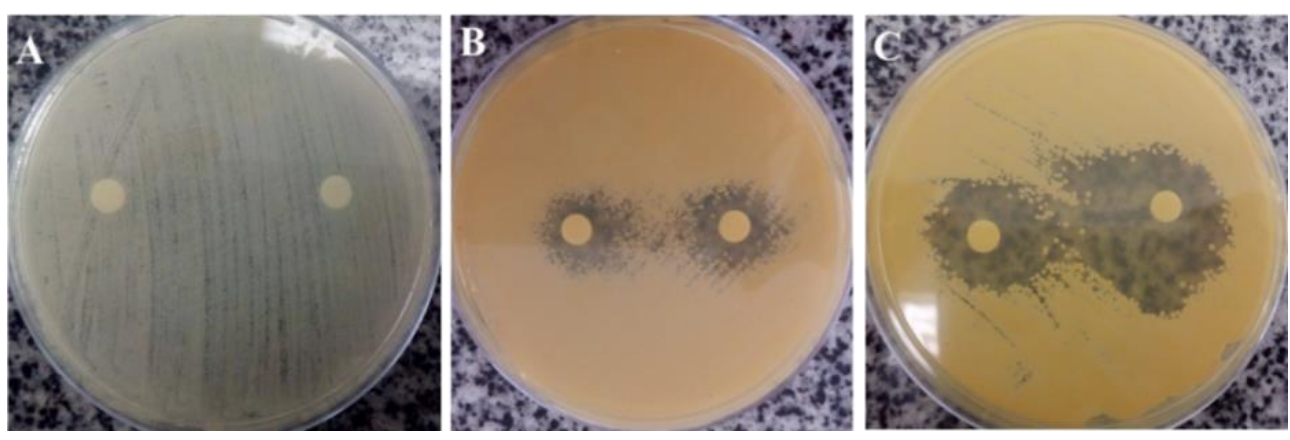

Figura 1. Amplitude da inibição do "pool" de contaminantes com $20 \mu \mathrm{L}$ de amostras em cada disco, em 144 horas de incubação a $37^{\circ} \mathrm{C}$. A: Hidrolato; B: Mistura de hidrolato e óleo essencial (1:1); C: Óleo essencial. Fonte: próprio autor.

Além de apresentar ação fungicida, estudos utilizando Mentha piperita também revelaram atividade antibacteriana de amplo espectro contra cepas como Escherichia coli, Salmonella typhius, Bacillus subtilis, Staphylococcus aureus, Pseudomonas aeruginosa, Staphylococcus epidermidis, Klebsiella pneumoniae, Proteus vulgaris e Salmonella entérica, de tal forma que este efeito inibitório é geralmente mais intenso para extratos aquosos e de acetato de etila (ANWAR et al., 2019).

Mahboubi e Kazempour (2014) relataram efeito de inibição contra Bacillus cereus, Enterococcus faecalis e Enterococcus faecium, Streptococcus mutans, Salmonella typhimurium, Pseudomonas aeruginosa, Shigella flexeneri, Shigella dysenteriae, Candida albicans e Aspergillus parasiticus. Da mesma forma que observaram um importante efeito sinérgico quando da associação entre o óleo de Mentha piperita e antibióticos.

\section{CONCLUSÃO}

Os dados de densidade e osmolaridade provenientes da caracterização físicoquímica dos produtos de extração do óleo essencial de Mentha piperita, obtidos por hidrodestilação, mostram que o hidrolato possui quantidades diminutas de biocompostos solúveis, diferindo consideravelmente da composição do óleo desta espécie. A caracterização de um meio ácido para ambas soluções, em especial àquela que apresenta maior relação de compostos orgânicos, aponta para a existência de ampla ocorrência de hidrólise de ésteres, potencializada principalmente pelo longo processo de extração em que 
a água em excesso fica submetida a altas temperaturas e em contato direto com o óleo.

Os testes de avaliação da atividade antimicrobiana mostram que tanto o óleo essencial puro quanto a mistura de hidrolato e óleo essencial na razão 1:1 apresentam propriedade antimicrobiana, principalmente verificada pela inibição do crescimento fúngico. A observação da atividade inibitória mesmo após um período de 144 horas para estas duas amostras, indica uma possibilidade viável na utilização do óleo essencial de Mentha piperita em combinação com o hidrolato como alternativa para o controle da proliferação de microrganismos, em especial leveduras, na conservação de alimentos.

\section{AGRADECIMENTO}

À Profa. Dra. Marina Ishii do Departamento Bioquímico-Farmacêutico (FCF/USP) pela utilização dos equipamentos para a análise físico-química.

\section{REFERÊNCIAS}

ANVISA. Agência Nacional de Vigilância Sanitária. Detecção e Identificação dos Fungos de Importância Médica: Módulo VII. Brasília, 2004.

ANWAR, F.; ABBAS, A.; MEHMOOD, T.; GILANI, A.; REHMAN, N. Mentha: A genus rich in vital nutra-pharmaceuticals - A review. Phytotherapy Research, 2019.

BEHN, H.; ALBERT, A.; MARX, F.; NOGA, G.; ULBRICH, A. Ultraviolet-B and photosynthetically active radiation interactively affect yield and pattern of monoterpenes in leaves of peppermint (Mentha x piperita L.). Journal of Agricultural and Food Chemistry, v. 58, p. $7361-7367,2010$.

CARRETTO, C. F. P.; ALMEIDA, R. B. A.; FURLAN, M. R.; JORGE, A. O. C.; JUNQUEIRA, J. C. Antimicrobial activity of Mentha piperita L. against Candida spp. Brazilian Dental Journal, v. 13, n. 1, p. 4-9, 2010.

COSTA, A. G.; CHAGAS, J. H.; PINTO, J. E. B. P.; BERTOLUCCI, S. K. V. Crescimento vegetativo e produção de óleo essencial de hortelã pimenta cultivada sob malhas. Pesq agropec bras, v. 47, n. 4, p. 534-540, 2012. 
FLEISHER, A.; FLEISHER, Z. Water-Soluble Fractions of the Essential Oils. Perfumer \& Flavorist, v. 16, p. 37-41, 1991.

FRANZENER, G.; MARTINEZ- FRANZENER, A. S.; STANGARLIN, J. R.; CZEPAK, M. P.; SCHWAN-ESTRADA, R. F.; CRUZ, M. E. S. Atividades antibacteriana, antifúngica e indutora de fitoalexinas de hidrolatos de plantas medicinais. Semina: Ci Agrárias, v. 28, n. 1, p. 29-38, 2007.

GUPTA, A. K.; MISHRA, R.; SINGH, A. K.; SRIVASTAVA, A.; LAL, R. K. Genetic variability and correlations of essential oil yield with agro-economic traits in Mentha species and identification of promising cultivars. Industrial Crops and Products, v. 95, p. 726-732, 2017.

HARE, J. M. Sabouraud agar for fungal growth. In: Laboratory Protocols in Fungal Biology: Current Methods in Fungal Biology, p. 211-216, 2012.

HOARE, J.; WILSON, S. Guia Completo de Aromaterapia: um curso estruturado para alcançar a excelência profissional, São Paulo: Pensamento, 2010.

JARRETT, L.; SONNENWIRTH, A. C. Gradwohl's and parasitic infections, 7. ed. American Public Health Association, Washington, DC, 1980.

KALEMBA, D.; SYNOWIEC, A. Agrobiological interactions of essential oils of two menthol mints: Mentha piperita and Mentha arvensis. Molecules, v. 25, n. 1, p. 59, 2019.

MACHIANI, M. A.; JAVANMARD, A.; MORSHEDLOO, M. R.; MAGGI, F. Evaluation of yield, essential oil content and compositions of peppermint (Mentha piperita L.) intercropped with faba bean (Vicia faba L.). Journal of Cleaner Production, 171, p. 529-537, 2018.

MAHBOUBI, M.; KAZEMPOUR, N. Chemical composition and antimicrobial activity of peppermint (Mentha piperita L.) Essential oil. Songklanakarin J. Sci. Technol. v. 36, n.1, p. 83-87, 2014.

MARCHESE, J. A.; FIGUEIRA, G. M. O uso de tecnologias pré e pós-colheita e boas práticas agrícolas na produção de plantas medicinais e aromáticas. Rev.Bras.PI.Med., v. 7, n. 3, p. 86-96, 2005. 
MEDEIROS, V. P. B.; SILVA, G. S.; LIMA, E. O.; PEREIRA, F. O. Identificação da microbiota fúngica anemófila em uma indústria de polpas de frutas e susceptibilidade antifúngica a terpenos. Rev Inst Adolfo Lutz, v. 74, n. 3, p. 266-273, 2015.

MOHAN, S. K. Beyond bacteria: Interpreting fungal elements in the Gram stain. Clinical Microbiology Newsletter, v. 26, n. 14, p. 108-112, 2004.

MONTEIRO, R. Desenvolvimento de Menta e Produção de Óleo Essencial Sob Diferentes Condições de Manejo. 2009. 81f. Dissertação de Mestrado - Universidade Federal do Paraná. Curitiba, 2009.

OLIVEIRA, A. R. M. F.; JEZLER, C. N.; OLIVEIRA, R. A.; MIELKE, M. S.; COSTA, L. C. B. Determinação do tempo de hidrodestilação e do horário de colheita no óleo essencial de menta. Horticultura Brasileira, v. 30, p. 155-159, 2012.

RAJESWARA RAO, B. R.; ADINARAYANA, G.; KUMAR, A. N.; RAJPUT, D. K.; SYAMASUNDAR, $\mathrm{K}$. V. Chemical-profile variations in essential oils isolated from lemongrass (Cymbopogon flexuosus) biomass and condensate wastewater by re-distillation and solvent extraction techniques. Journal of Essential Oil Research, v. 28, n. 6, p. 557-564, 2016.

SINGH, R.; SHUSGNI, M. A. M.; BELKHEIR, A. Antibacterial and antioxidant activities of Mentha piperita L. Arabian Journal of Chemistry, v.8, n.3, p.322-328, 2015.

SOLANKI, K. P.; DESAI, M. A.; PARIKH, J. K. Improved hydrodistillation process using amphiphilic compounds for extraction of essential oil from java citronella grass. Chemical pap, v. 74, p. 145-156, 2019.

SOUZA, D. R.; SOUZA, G. A.; ARAUJO, L. M. P.; BEZERRA, V. S.; MARQUES, R. B. Efeitos tóxicos dos fungos nos alimentos. Revinter, v.10, n.2, p.73-84, 2017.

SOUZA, W. P.; QUEIROGA, C. L.; SARTORATTO, A.; HONÓRIO, S.L. Avaliação do teor e da composição química do óleo essencial de Mentha piperita (L.) Huds durante o período diurno em período hidropônico. Rev. Bras. PI. Med., v.8, n.4, p. 108-111, 2006. 
STERRETT, F. S. The Nature of Essential Oils. Journal of Chemical Education, v. 39, n. 4, p. 203-206, 1962.

VAN-UDEN, N. Temperature Profiles of Yeasts. Advances in Microbial Physiology, v. 25, p. 195-251, 1985.

WATANABE, C.H.; NOSSE, T.M.; GARCIA, C.A.; PINHEIRO POVH N. Extração do óleo essencial de menta (Mentha arvensis L.) por destilação por arraste a vapor e extração com etanol. Rev Bras PI Med, v.8, n.4, p.76-86, 2006.

*Autor para correspondência:

Évellin do Espirito Santo

Endereço: Universidade de São Paulo, Campus Cidade Universitária, Av. Prof Lineu Prestes, 580, Bairro Butantã, 05508-000, São Paulo/SP, Brasil.

Email: evellin.santo@gmail.com

Recebido: 28/08/2020 Aceite: 30/09/2020 